\title{
The Role of Astrocytes in Neuroprotection after Brain Stroke: Potential in Cell Therapy
}

\author{
Andrea Becerra-Calixto and Gloria P. Cardona-Gómez* \\ Cellular and Molecular Neurobiology Area, Group of Neuroscience of Antioquia, School of Medicine, Sede de Investigación \\ Universitaria (SIU), University of Antioquia, Medellin, Colombia
}

Astrocytes are commonly involved in negative responses through their hyperreactivity and glial scar formation in excitotoxic and/or mechanical injuries. But, astrocytes are also specialized glial cells of the nervous system that perform multiple homeostatic functions for the survival and maintenance of the neurovascular unit. Astrocytes have neuroprotective, angiogenic, immunomodulatory, neurogenic, and antioxidant properties and modulate synaptic function. This makes them excellent candidates as a source of neuroprotection and neurorestoration in tissues affected by ischemia/reperfusion, when some of their deregulated genes can be controlled. Therefore, this review analyzes pro-survival responses of astrocytes that would allow their use in cell therapy strategies.

Keywords: astrocytes, cell therapy, neuroprotection, cerebral ischemia, excitotoxicity

\section{OPEN ACCESS}

Edited by:

Marina Guizzetti,

Oregon Health \& Science University,

USA

Reviewed by:

Clare Howarth,

University of Sheffield, UK

Philip Forsyth Copenhaver, Oregon Health \& Science University,

USA

*Correspondence:

Gloria P. Cardona-Gómez

patricia.cardonag@udea.edu.co

Received: 18 August 2016

Accepted: 14 March 2017

Published: 03 April 2017

Citation:

Becerra-Calixto A and

Cardona-Gómez GP (2017) The Role of Astrocytes in Neuroprotection after

Brain Stroke: Potential in Cell

Therapy. Front. Mol. Neurosci. 10:88.

doi: 10.3389/fnmol.2017.00088

\section{INTRODUCTION}

Until a few decades ago, astrocytes were considered to be glial support cells, with roles limited to providing trophic, metabolic, and structural support to the neurons. Currently, multiple investigations have revealed the multi-faceted role of astrocytes in cerebral parenchymal homeostasis, which depends on intercellular communication (Dallérac et al., 2013). It has been widely reported that astrocytic cells play protective roles in the nervous system, characterized by the ion buffering (Walz, 2000; D'Ambrosio et al., 2002), the uptake and synthesis of neurotransmitters (Danbolt, 2001; Kaczor et al., 2015), controlling cerebral blood flow (Abbott et al., 2006;

Abbreviations: AGC, aspartate/glutamate carrier; ALS, amyotrophic lateral sclerosis; Ang, angiopoietin; APCs, antigen presenting cells; $\beta \mathrm{A}, \beta$-amyloid; $\mathrm{BBB}$, blood-brain barrier; $\mathrm{BDNF}$, brain-derived neurotrophic factor; bFGF, basic fibroblast growth factor; BMEC, brain micro-endothelial cells; CDK5, cyclin-dependent kinase 5; CNTF, ciliary neurotrophic factor; Cx43, connexin-43; DAB, 1,4-dideoxy-1,4-imino-D-arabinitol; DCX, doublecortin; DG, dentate gyrus; EAATs, excitatory amino acid transporters; ECs, endothelial cells; EPCs, endothelial progenitor cells; GDNF, glial-derived neurotrophic factor; GFAP, glial fibrillary acidic protein; GLAST, glutamate aspartate transporter; GLT-1, glutamate transported 1; GMCSF, glial-monocyte colony stimulating factor; GS, glutamine synthetize; GSH, glutathione; GSK3, glycogen synthase kinase 3; HMGB1, high-mobility group box 1; IGF, insulin growth factor; IL, interleukin; iPSCs, induced pluripotent stem cells; KO, knockout; LPS, lipopolysaccharides; LTP, long-term potentiation; MAPs, microtubule-associated proteins; MSC, mesenchymal stem cells; NH4, ammonium; NMDAR, $N$-methyl-D-aspartate receptor; NO, nitric oxide; NPs, neural progenitors; Nrf2, nuclear factor erythroid 2-related factor 2; NS, neurosphere; NSCs, neural stem cells; NT-4, neurotrophin 4; NVU, neurovascular unit; OGD/R, oxygen glucose deprivation/reperfusion; PECAM1, platelet and endothelial cell adhesion molecule 1; Pfkfb3, 6-phosphofructose-2-kinase/fructose-2,6-biphosphatase-3; PRRs, pattern-recognition receptors; RAGE, receptor for advanced glycation end products; RhoA and Rac1, Rho family of GTPase members; ROS, reactive oxygen species; S100b, calcium-binding protein B; SGL, subgranular layer; siRNA, small interference Ribonucleic acid; SVZ, subventricular zone; TGF $\beta$, transforming growth factor- $\beta$; TLRs, Toll-like receptors; TRAIL, TNF-related apoptosis-inducing ligand; VEGF, vascular endothelial growth factor. 
Newman, 2015), transport of water (Zeng et al., 2007; Kitchen et al., 2015), release of antioxidant substances (Pentreath and Slamon, 2000), and immunomodulation (Dong and Benveniste, 2001). Also, it has recently been described that astrocytes are involved in adult neurogenesis (Mori et al., 2005; Buffo et al., 2008; Duan et al., 2015; Nato et al., 2015), making the astrocyte a highly complex cell (Figure 1A). However, under pathological conditions, hyperreactive astrocytes exacerbate its heterogeneous functions, presenting an opposite role that, contribute to the central nervous system (CNS) disbalance (Figure 1B).

Astrocytes are characterized as having a stellate morphology, which changes to a reactive state under stress and degenerative conditions. This individual morphology is directly related to the expression of glial fibrillary acidic protein (GFAP; Lebkuechner et al., 2015); the upregulation of GFAP depends on the nature of damage, the distance between the astrocyte and the site of injury, and the time after injury (Eng et al., 2000; Sun et al., 2010). On the other hand, it has been reported that reactive astrogliosis and astrocyte proliferation also are neuroprotective, because on this condition the glial cells can to provide factors that promote cell survival against severe injury or degeneration (Mohn and Koob, 2015; Figure 1B). However, under ischemia context, for example, the interruption of blood supply to the brain results in deprivation of oxygen and glucose and the consequent reduction of the energy supply. This process leads to excessive accumulation of ions and the deregulation of several signaling pathways, overloading the buffering role of the astrocytes, which go to favor of the activation of catabolic processes mediated by proteases, lipases, and nucleases, which disrupt neuronal function and lead to cell death (Lipton, 1999). Therefore, a better understanding of the astrocyte's mechanism for triggering cell cytotoxicity or keeping its neuroprotective activity would help to control the parenchyma dyshomeostasis and to prevent progressive brain degeneration. In this review, we will focus on the cellular mechanism of the astrocytes for providing neuroprotection, and we will propose that the downregulation of proteins associated with pathological states could allow maintenance of its survival activity in the injured tissue, recovering its integrity and function.

\section{ASTROCYTES IN NEURODEGENERATION AFTER BRAIN STROKE}

Astrocytes, unlike neurons, are less vulnerable to glutamate excitotoxicity during a brain stroke, however, induces its proliferation and increase GFAP levels in a process termed reactive astrogliosis (Sofroniew and Vinters, 2010). Susarla et al. (2014) reports that reactive protoplasmic astrocytes in the cortex began to proliferate within 3-5 days after injury, and the half of them reentering to the cell cycle up to a week later. Reactive astrogliosis have been related widely as a pathological hallmark of altered CNS tissue (Igarashi et al., 1999; Chen and Swanson, 2003; Rodríguez et al., 2009; Sofroniew and Vinters, 2010; Robel et al., 2015; Sofroniew, 2015). Which are generally found in areas surrounding severe focal lesions, and it is characterized by astrocyte proliferation and a considerable extension of processes beyond the previous domains of individual astrocytes (Swanson et al., 2004). These changes can result in reorganization of tissue architecture without the formation of dense narrow and compact barriers, as in glial scars (Sofroniew and Vinters, 2010; Shimada et al., 2011). The glial scar is the most characteristic profile of the reactive astrogliosis. In a severe state, a compact glial scar formation, includes changes associated with milder forms, with upregulation of GFAP and other genes, and pronounced hypertrophy of cell bodies and processes, interacting with other type of glial cells (Silver and Miller, 2004; Sofroniew and Vinters, 2010). In addition, glial scar formation has been considered an inhibitor of axon regeneration, as a maladaptive phenomenon that unavoidably causes neurotoxicity, inflammation, or chronic pain, supported by the increase of pro inflammatory factors levels, as interleukins $1 \beta, 6,10$ (IL-1, IL-1 $\beta$, IL-6, IL-10), interferon- $\gamma$ (IFN- $\gamma$ ), transforming growth factor- $\beta$ (TGF $\beta$ ) (Rostworowski et al., 1997; Suzumura et al., 2006), reactive oxygen species (ROS), nitric oxide (NO), glutamate, calcium-binding protein B (S100b) (Piani et al., 1993; Pekny and Nilsson, 2005; Mori et al., 2009; Liu et al., 2011; Shen et al., 2012; de Pablo et al., 2013; Jiang et al., 2013). Also, producing molecules associated to infection and metabolic disorders, which exacerbate the disease, as Toll-like receptors (TLRs), lipopolysaccharides (LPS), $\beta$-amyloid ( $\beta$ A; $\mathrm{Hu}$ and Van Eldik, 1999; Caso et al., 2007; Gorina et al., 2011; Sofroniew, 2014), and ammonium (NH4; Lichter-Konecki, 2008). In brain stroke, reactive astrocytosis is related with "chronic cystic infarcts," characteristic finding in a cortical cystic infarct is the preservation of the relatively paucicellular molecular layer overlying it and located immediately beneath the meninges, which then develops a dense collection of astrocytes, usually with gemistocytic phenotype. Astrocytic processes usually traverse the cystic cavity left by the death of brain tissue, in a delicate meshwork, with persistence of foamy histiocytes among and influx of polymorphonuclear leukocytes and macrophages (Sofroniew and Vinters, 2010). In the counterpart, there is a specific process of reactive astrogliosis that has beneficial functions and does not do harm. As describe below, many studies using transgenic and experimental animal models provide evidences that reactive astrocytes protect CNS tissue by the same mechanisms that astrocytes has homeostatic functions, as well as the induction of neurogenesis, regulation of immune system and maintain the blood-brain barrier (BBB) integrity. This evidence changes astrocytosis, from being viewed as harmful to being neuroprotective, while pathogenic expressions are being regulated (Figure 1).

\section{SUPPORT OF ENERGY METABOLISM AND ANTIOXIDANT EFFECTS OF ASTROCYTES}

Brain is a high energy-consuming organ; for these reason, brain cells can efficiently to utilize various energy substrates in addition 


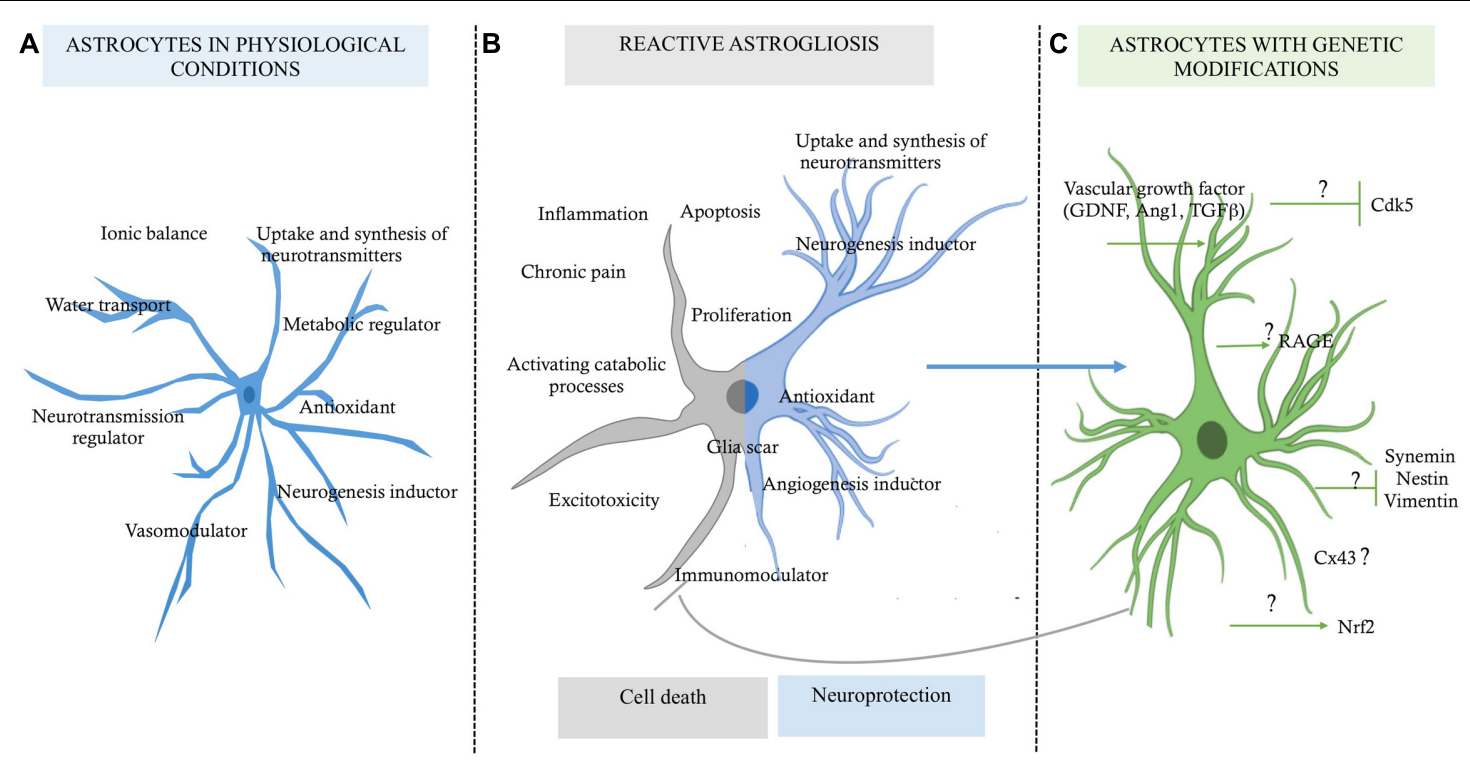

FIGURE 1 | Role of astrocytes in a micro-environment dependent-mode. (A) Functions of the astrocytes in physiological conditions, which are in favor of the homeostasis of the nervous tissue. (B) Reactive astrocytosis, which has a double function highly discussed, one for cell death and one for pro-neuroprotection probably in a context dependent-mode. (C) Astrocytes with genetic modifications by reduced expression of some upregulated genes, which would allow preserve them as a neuroprotective source for promoting neuronal survival; although the mechanism of how they could maintain this state of neuroprotection for longer time is still unknown (?).

to glucose, including lactate, pyruvate, glutamate, and glutamine (Bé Langer et al., 2011). Neurons and astrocytes present different metabolic profiles, although are complementary. However, astrocytes are reported that has an exclusive characteristic of support the neuron energetic needs. One of recently reported is the lactate metabolism represent an import pathway of glucose metabolism (Lovatt et al., 2007). Astrocytes present a high glycolytic rate, so enzymes as 6-phosphofructose2-kinase/fructose-2,6-bisphosphatase-3 (Pfkfb3) are related to the activation of glycolytic pathway, involving the lactate as result of glucose oxidation. Those enzymes have high expression levels in astrocytes, but it is absent in neurons due to constant proteasome degradation (Herrero-Mendez et al., 2009). Another characteristic of astrocytes is the low expression levels of aspartate/glutamate carrier (AGC), which is a component of maleate aspartate shuttle, which operates the transfer of reducing equivalents from the cytosol to mitochondria (Berkich et al., 2007). In this context the conversion of derived pyruvate to lactate in cytosol ensure the maintenance of high $\mathrm{NAD}^{+} / \mathrm{NAD}$ ratio, being essential to sustain a high glycolytic rate (Bé Langer et al., 2011).

Another characteristic of astrocytes is the glycogen metabolism. Glycogen is the largest energy reserve of the brain and it can be rapidly metabolized under anaerobic conditions. Glycogen has been found to be almost exclusively localized in astrocytes in the adult brain (Brown, 2004), this characteristic suggest the close interaction between astrocytes and neurons. Brown et al. (2005) demonstrated that increasing astrocytic glycogen stores preserves the neuronal function and viability under limited conditions of energy, such as in hypoglycemia. Also, neuron-glia metabolic coupling involves glycogen content under the dynamic control of neurotransmitters and the neural functions (Brown et al., 2005). Gibbs et al. (2006) demonstrated that pharmacological inhibition of glycogenolysis in astrocytes using 1,4-dideoxy-1,4-imino-D-arabinitol (DAB), a potent inhibitor of glycogen phosphorylase, interrupts memory consolidation in young chickens in a bead discrimination learning task. Also, the role of astrocytic glycogen-derived lactate in long term memory formation, and for the in vivo maintenance of long-term potentiation (LTP) of synaptic strength in the mammalian brain (Suzuki et al., 2011). These findings support that the astrocytes play a major role in the metabolic maintenance of neurons, which are directly related to its functionality.

Additional characteristic of astrocytes is the role in the synthesis, re-uptake or recycling of neurotransmitters. It is widely known that astrocytes rapidly remove neurotransmitters that that are released in the synaptic cleft. This function is an essential process to guarantee the effective synaptic process and the maintenance of neuronal excitability. One of most important excitatory neurotransmitter is glutamate, its overstimulation is highly toxic for neurons, the way that astrocyte uptake the glutamate is through specific glutamate transporters named glutamate transported 1 (GLT-1), and glutamate aspartate transporter (GLAST) (Bak et al., 2006) in a glutamate-glutamine cycle (Shen et al., 1999; Danbolt, 2001). Astrocytes are responsible for replenishment of brain glutamate and are the only cell type in the brain which express pyruvate carboxylase, an enzyme involved in the anaplerotic pathway 
in the brain, effectively allowing them to synthesize glutamate from glucose (Bélanger and Magistretti, 2009; Bé Langer et al., 2011).

In brain stroke, the decrease of oxygen and glucose, produce an alteration of the glutamate levels, producing excitotoxicity in neurons. The evidence shows that GLAST and GLT-1 are downregulated shortly following the insult, which then precipitates glutamate-mediated excitotoxic conditions (Yi and Hazell, 2006; Zou et al., 2010). Also, Zou et al. (2010) demonstrated that glutamine synthetize (GS) inhibition in astrocytes significantly impaired glutamate uptake, suggesting that GS in astrocytes may represent a novel target for neuroprotection against neuronal dysfunction. Liang et al. (2008) compared the expression of excitatory amino acid transporters (EAATs) in astrocytes and microglia, analyses demonstrated that astrocytes express a much larger amount of membrane localized EAATs than microglia. Astrocytes prevented excito-neurotoxicity by the reduction of exogenous glutamate, whereas microglia did not. Conversely, activated microglia released an excess of glutamate that induced excitotoxic neuronal death. Astrocytes rescued neurons from microglial glutamate-induced death in a ratio-dependent manner. Inhibition of EAATs abolished glutamate uptake and the neuroprotective effect of astrocytes, but it did not alter microglial neurotoxic or neuroprotective effects. These results revealed that astrocytic EAATs can counteract microglial glutamate-induced neuronal death, whereas microglial EAATs are not involved in the toxicity and protection of astrocytes and microglia in a co-culture system (Liang et al., 2008). Those findings suggest that maintaining the expression and regulation of the transporters in astrocytes would be a potential source of clinical intervention treatment following brain ischemia.

On the other hand, astrocytes are involved in the defense against oxidative stress. Stroke produces several factors that contribute to increased brain vulnerability to oxidative stress, including its high rate of oxidative energy metabolism (an inevitable process generating ROS), and its high unsaturated fatty acids content (which are prone to lipid peroxidation; Dringen, 2000). Glutathione (GSH) is the most abundant antioxidant molecule in the brain, which acts directly as ROS scavenger or can be used as substrate for glutathione peroxidase (Dringen, 2000). GSH is regenerated for action of glutathione reductase, using $\mathrm{NADPH}$ as an electron donor; this process is essential for the maintenance of GSH in its reduced form (Bé Langer et al., 2011). NADPH is more abundant in astrocytes than in neurons, and astrocytes have a higher basal activity rate and a better capacity to stimulate this pathway in response to oxidative stress (Garcia-Nogales et al., 2003). Also, astrocytes release the antioxidant molecule ascorbic acid in response to glutamatergic activity. This ascorbic acid is taken up by neurons and modifies the local energy metabolism by inhibition of glucose consumption and increased uptake of lactate (Castro et al., 2009). Moreover, there is another molecule that is expressed in astrocytes and it has neuroprotective functions, the redox-sensitive transcription factor, named nuclear factor erythroid 2-related factor 2 (Nrf2) activation. Nrf2, is a redox-sensitive transcription factor, that coordinates expression of genes required for free radical scavenging, detoxification of xenobiotics, and maintenance of redox potential (Shih et al., 2005). In stroke, Nrf2 pathway is activated in both in vitro and in vivo ischemic models. In addition, to mediate self-defense in neurons, Nrf2 also actively regulates the expression of cytoprotective enzymes in other cell types within the neurovascular unit (NVU), including astrocytes and endothelial cells (ECs), and thus supports neuronal function and survival through cell-cell interaction (Yang et al., 2016).

A large body of experimental evidence suggests that astrocytes have a greater metabolic plasticity than neurons. A striking example is the differential response of astrocytes and neurons following the inhibition of mitochondrial respiration induced by NO. Astrocytes respond to NO with an increase in glucose metabolism through the glycolytic pathway, limiting the fall in ATP levels and preventing apoptosis. In neurons, however, this response does not seem to be present, and a similar NO challenge causes a massive ATP depletion, leading to apoptosis (Almeida et al., 2001; Bé Langer et al., 2011).

\section{ASTROCYTES MODULATE THE IMMUNE RESPONSE}

In ischemic conditions, reactive astrocytes are involved in the immune response because astrocytes mediate and propagate inflammatory signals in the brain, influencing various physiological and behavioral responses. Astrocytes were the first CNS cell type where was demonstrated the expression of class II major histocompatibility complex (MHC) molecules (Dong and Benveniste, 2001). MHC II is a molecule that play a critical role in the induction of immune responses through the presentation of processed antigens to CD41 T-helper cells, this molecule is normally expressed on professional antigen presenting cells (APCs), such as B cells, macrophages, dendritic cells, and other cell types, including astrocytes. MHC II expression allow to astrocytes can be regulated by cytokines, neurotransmitters, and neuropeptides (Dong and Benveniste, 2001). Although, the MHC is involve in an exacerbated inflammation response in astrocytes. Also, cytokines have neuroprotective and neurotrophic roles required for neurodevelopment and maintenance of normal CNS function (Maier et al., 2005; Morganti-Kossmann et al., 2007).

As mentioned before astrocytes are also capable of synthesizing cytokines and chemokines; they express patternrecognition receptors (PRRs), such as TLRs, scavenger receptors, and complement proteins. There is evidence that astrocytes play a complex and a dual role in the local regulation of immune reactivity. Astrocytes are resistant to apoptosis induced after inflammation by death receptors, which is known as apoptosis antigen 1 and tumor necrosis factor (TNF)-related apoptosisinducing ligand (FAS, TRAIL), indicating that these cells are well prepared to survive under inflammatory insults (Farina et al., 2007). Neurotrophic mediators such as glial-monocyte colony stimulating factor (GM-CSF), vascular endothelial growth factor 
(VEGF), neurotrophin 4 (NT-4), and ciliary neurotrophic factor (CNTF) can activate TLRs in astrocytes and stimulate restorative pathways (Bsibsi et al., 2006).

In an ischemic condition, activated microglia and activated astrocytes (reactive astrogliosis), might to secret products exerting neuroprotective actions during glial scar formation. It has been demonstrated that reactive astrocytes and microglia can to demarcate the damaged area and to limit the leukocyte extravasation, promoting $\mathrm{BBB}$ repair and neuronal survival (Farina et al., 2007). Some cytokines, such as IL-1 and IL-6, related to pathological inflammatory responses in different processes might also behave as a mediator of neuroprotection. Some studies have demonstrated that deletion of IL- 6 and IL-1 $\beta$ increases $\mathrm{BBB}$ permeability and decreases the production of neurotrophic factors such as CNTF and insulin growth factor (IGF), indicating that cytokine-induced astrogliosis following trauma is important to restore the integrity of the BBB and to repair the lesion (Herx et al., 2000; Mason et al., 2001). Several in vitro data demonstrate that cytokines such as IL-1, IL-6, and TNF support the production of neuroprotective mediators. Other proteins implicated in inflammation response, are the chemokines, which facilitate the mobilization of leukocytes and polymorphonuclear cells. Additionally, these chemokines contribute to the migration of neural progenitors in the developing brain (Tran and Miller, 2003) and toward areas of brain injury (Belmadani et al., 2006; Farina et al., 2007). These characteristics make the astrocyte a cell highly sensitive, inductor and possibly regulator of immune responses, being one of main important characteristic in an ischemic context, because the chronicity of inflammation depends on the degree of tissue damage and exacerbation of the injury.

\section{NEUROPROTECTIVE ROLE OF ASTROCYTES}

Under a loss of cerebral parenchymal integrity, astrocytes would play a protective role whether is possible to control its reactivity (Li et al., 2008), which would allow maintaining the homeostatic functions. In particular, astrocytes are involved in a large number of key processes in the nervous system, including crucial roles in regulating vascular tone; removing excess glutamate in synaptic cleft thus limiting the neuronal activity; promoting synaptogenesis; releasing neurotrophic factors; secreting different antioxidants and responding to the release of pro and anti-inflammatory molecules (Sofroniew and Vinters, 2010). Astrocytes have also been shown to regulate the blood flow during neuronal activity, via the release of vasoactive substances such as NO, products derived from activity of epoxygenase, ATP and cyclooxygenase, activation of phosphatidylinositol 3kinase $(\mathrm{PI} 3 \mathrm{~K})$ and calcium waves propagated from the neuron to astrocyte and endothelium (blood vessel cells; Abbott et al., 2006), and regulate potassium ions $\left[\mathrm{K}^{+}\right]$resulting from synaptic activity (Benarroch, 2005; Bean, 2007). Concomitant with the ionic and metabolic regulation by astrocytes, these cells may regulate the release of neurotrophic factors, which facilitate neuronal survival and angiogenesis (Goss et al., 1998; Rz et al., 1999; Jiang et al., 2013). These neurotrophic factors have varied effects on neurons, ECs, microglia, leukocytes, and neural stem cells (NSCs) (Schwartz and Nishiyama, 1994; Igarashi et al., 1999; Swanson et al., 2004; Oliveira et al., 2013; Götz et al., 2015).

\section{ASTROCYTIC INVOLVEMENT IN ADULT NEUROGENESIS}

The adult neurogenesis in the adult mammalian brain has been described in two specific zones, named neural niches; the subgranular layer (SGL) of the dentate gyrus (DG) of the hippocampus and the subventricular zone (SVZ) in the lateral wall of the lateral ventricle (Seri et al., 2001). The primary precursors in the SVZ, the other germinal region of the adult brain, have been identified as having the characteristics of astrocytes and expressing GFAP (Laywell et al., 2000). The reports are suggested that some of these cells can maintain a neurogenic potential and act as NSCs (Mori et al., 2005; Magnusson and Frisén, 2016). These cells can divide and generate new neurons under normal conditions or after the chemical removal. Seri et al. (2001) found GFAP $^{+}$cells called type D cells are derived from the astrocytes and probably function as a transient precursor in the formation of new neurons. However, deeper research is needed to understand the transdifferentiation of astrocytes in NSC or a transient state as trophic source without a definitive commitment to other specific linage.

After brain ischemic event, astrocytes acquire stem cell hallmarks mainly in vitro conditions; in vivo is still controversial because the multipotentiality is depending of growth factors (Götz et al., 2015). In vitro assays have shown that some of the reactive astrocytes in the traumatic or ischemic brain acquire neurosphere (NS)-forming ability, multipotency and long-term self-renewal, while others remain within their astrocyte lineage in vivo (Götz et al., 2015). Jiang et al. (2013) found two different types of cells that are NPC-astroglial cells (NPC-Astros) and Olig2PC-astroglial cells (Olig2PC-Astros), respectively. When was grafted into brains subjected to global ischemia, Olig2PCAstros exhibit neuroprotective effects and improved behavior (Jiang et al., 2013). On other hand, Ohab et al. (2006) reported that stroke induces neurogenesis from a GFAP-expressing progenitor cell in the SVZ and migration of newly born neurons into a unique neurovascular niche in peri-infarct cortex. Also, Faiz et al. (2015) described that reactive astrocytes can convert to multipotent NSCs capable of NS formation and multilineage differentiation in vitro. Moreover, they reported that SVZ NSCs give rise to a reactive astrocytes subpopulation in the cortex that contribute to astrogliosis and scar formation and they found that these astrocytes in SVZ can be converted to neurons in vivo by forced expression of Ascll (Faiz et al., 2015).

Astrocytes also preserve the function of the hippocampal neural niches, where adult neurogenesis plays an important role. Sultan et al. (2015) found that astrocytes can allow the 
synaptic integration of adult-born hippocampal neurons, allowing local dendritic spine maturation, which is necessary for the $N$-methyl-D-aspartate receptor (NMDAR)-dependent functional integration of newborn neurons. Moreover, Tao et al. (2015) demonstrated that some proteins as $\beta$-arrestin$1(\beta$-arr1) is secreted by astrocytes in DG and participates in adult hippocampal neurogenesis. The model of $\beta$-arr1 knockout (KO) astrocytes produced less NSs, and RNAsequencing revealed that $\beta$-arr1 KO DG astrocytes exhibit an aberrant gene expression profile of niche factors, including elevated transcription of Bmp2. Those results suggest that $\beta$-arr1-mediated nuclear signaling regulates the production of excretive factors derived from niche of astrocytes and expansion of neural precursors in DG, thus maintaining homeostasis of adult hippocampal neurogenesis (Tao et al., 2015). Additionally, Li et al. (2014), in an oxygen glucose deprivation/reperfusion (OGD/R) model, examined the levels of a damage-associated molecular-patterning molecule called high-mobility group box 1 (HMGB1) that is release by astrocytes. HMGB1 is critical for NSC/neuroprogenitors proliferation during brain development. The data demonstrate that HMGB1 released by OGD/R astrocytes promotes NSC proliferation through activation of the $\mathrm{PI} 3 \mathrm{~K} /$ protein kinase $\mathrm{B}$ (PI3K/Akt) signaling pathway (Li et al., 2014). These findings demonstrate that astrocytes are involved key roles in the generation of new neurons and maintenance, stimulation of neural niches after an ischemic event, and possibly in the regulation of adult neurogenesis in physiological conditions, which do them targets for isolation, study and cell therapeutic cells in this type of brain damage. However, some characteristic of NSC associate with astrocytes still is controversial, hence their neurogenic potential must be further explored.

\section{ANGIOGENESIS CAN BE MODULATED BY ASTROCYTES}

The appropriate supply of oxygen and glucose, as well as the appropriate secretion of metabolites by the brain, depends on close relationships between the vascular system, glial cells, and neurons. The interconnection between these cells is known as the NVU. Astrocytes have very close interactions with neurons and ECs, which composite the blood vessels. In this interaction participate various membrane proteins such as ion and water channels and receptors for growth factors and cytokines. Also, astrocytes and ECs can release neurotrophins, vascular growth factors, glucose, and amino acids, in order to generate stability and maintenance of the BBB (Abbott et al., 2006).

During brain ischemia, there is a series of events that compromise the integrity of the BBB. Glucose deficit and hypoxia trigger cellular stress, not only in neurons, but also in ECs and astrocytes. This cellular stress causes the release of oxygen, free radicals, and $\mathrm{NO}$; and also the production of pro-inflammatory cytokines that allow permeabilization of the $\mathrm{BBB}$ and therefore infiltration of leukocytes, which exacerbate the inflammatory response and increase the triggering of apoptotic signals (Chodobski et al., 2011). In this condition, signaling between astrocytes, pericytes, and endothelium become disrupted. Hence, repairing the gliovascular system include a crosstalk between astrocytes and pericytes (Bell et al., 2010). Astrocytes are known to release thrombospondin-1, which is a major regulator of synaptic maturation (Christopherson et al., 2005). Reactive astrocytes also release tissue plasminogen activator, which may be required for recovering neurons to remodel their dendritic arbors (Xin et al., 2010). Indeed, several studies have suggested that the therapeutic benefits of stem cell therapies may depend in part on the ability of astrocytes to amplify the effects on neuronal remodeling (Shen et al., 2010). Astrocytes and cerebral ECs secrete many trophic factors that support oligodendrocyte precursor cells (Arai and Lo, 2009). And after injury, VEGF-mediated endothelial recovery is linked with the proliferation and migration of oligodendrocyte precursor cells (Le Bras et al., 2006; Hayakawa et al., 2011).

Moreover, astrocytes has different mechanism to modulate cytotoxic response, as inducing angiogenesis necessary for the reestablishment of the blood circulation after brain ischemia. This mechanism consist of the secretion of a range of chemical agents and glia-derived factors, including TGF $\beta$, glial-derived neurotrophic factor (GDNF), basic fibroblast growth factor (bFGF), angiopoietin 1 (Ang1) (Igarashi et al., 1999; Wuestefeld et al., 2012). These factors can stimulate the production of new blood vessels and the proliferation of endothelial progenitor cells (EPCs; Cross et al., 2001; Babaei et al., 2003). Different studies using co-cultures of brain ECs and astrocytes have demonstrated that astrocytes express functional receptors for a high proportion of agents that mobilize ECs (Abbott et al., 2006). Additionally, reactive astrocytes can release factors, as HMGB1, VEGF, Ang1, and angiotensin 2 (Ang2) (Hayakawa et al., 2012; Duan et al., 2015; Shen et al., 2016); which promotes EPC-mediated neurovascular remodeling during stroke recovery (Krum and Rosenstein, 1998). Hayakawa et al. (2011, 2012), found that blocking the "receptor for advanced glycation end products" (RAGE) increased the reactive astrocyte and ECs significantly decreased EPC-endothelial adherence. RAGE is a multi-ligand receptor that propagates cellular dysfunction in several inflammatory disorders, tumors and diabetes, and it is upregulated at sites where its ligands are accumulated; being low expressed in normal tissues. Also, it has been reported that RAGE may play a dual role in the inflammatory response, because interaction of RAGE on leukocytes or ECs with its ligands results in cellular activation involving the transcription factor nuclear factor kappa-light-chain-enhancer of activated B cells (NFkappaB). On the other hand, RAGE on ECs may function as an adhesive receptor that directly interacts with leukocyte ss2integrins (Chavakis et al., 2004). So, through the upregulation of RAGE on affected ECs, the reactive astrocytes may augment EPC adherence and transmigration. This phenomenon may comprise a novel mechanism whereby crosstalk between reactive astrocytes and cerebral endothelium augments EPC responses for neurovascular recovery in damaged or diseased brain. In this order, RAGE is important in EPC induction and adherence 
that can play an important role in tissue vascularization and endothelium homeostasis after CNS damage by ischemia. Those findings suggest that astrocytes can partially regulate the effects of ischemia through a continue communication with NVU cells.

\section{POSSIBLE PROTEINS INVOLVED IN ASTROCYTES REACTIVITY AND REGENERATION}

As described above, astrocytes appear to possess a high potential for regeneration and neuroprotection following stroke, but also there are substantial evidence that has shown that astrocytes are cells that exacerbate ischemic injury (Figure 1B).

So, one possible paradigm of astrocyte reactivity control is the expression of GFAP. Increased GFAP expression is associated with hyperreactivity and altered morphology of the astrocyte. Also, connexin-43 $(\mathrm{Cx} 43)$ is a gap junction protein that is associated with reactive astrogliosis. In response to an acute needle stab wound in vivo, reactive astrocytes expressed $\mathrm{Cx} 43$ in the peripheral zone surrounding the injury site (Theodoric et al., 2012). In addition, intermediate filament proteins such as vimentin, nestin, and synemin are involved in the reactivity of astrocytes and are highly expressed in conditions of deprivation of oxygen and glucose, as well as in response to oxidative stress (de Pablo et al., 2013). However, the regulatory mechanisms underlying the development of reactive astrocytes during basal function and under neuroprotective hypertrophic function remain unknown.

Other protein involved in the reactive astrocyte that has been poorly studied is cyclin-dependent kinase 5 (CDK5). This protein is highly expressed in post-mitotic neurons. In the brain, it is involved in functions such as neuritogenesis, synapse formation, synaptic transmission, and the assembly, organization and stabilization of the axonal cytoskeleton, and it allows the activation of apoptosis. Its substrates are mainly neurofilaments, microtubule-associated proteins (MAPs) and elements of the axonal cytoskeleton (Grant et al., 2001). Although CDK5 is generally studied in neurons, it is not exclusively expressed in those cells; some reports show that CDK5 is active in nonneuronal cells such as astrocytes, ECs, and blood cells (Liebl et al., 2011). In astrocytes, CDK5 has a very important activity in the process of elongation and reactivity. He et al. (2007) report that p35, GFAP, and CDK5 can form immunocomplexes in primary cultures of astrocytes. p35 is activated when the astrocyte is subjected to stress caused by injury, generating active CDK5. Astrocytes treated with roscovitine, a CDK5 inhibitor, show reorganization of tubulin and GFAP (He et al., 2007). In addition, other findings have shown that CDK5 plays a significant role in the regulation of EC proliferation (Liu et al., 2008). Therefore, CDK5 dysregulation in excitotoxic processes can generate changes not only in neurons but elsewhere in the NVU, which is highly regulated by astrocytes. This in turn leads to increased reactivity and alteration of each of the components and the integrity of the NVU and results in brain dysfunction.

\section{COULD GENETICALLY MODIFIED ASTROCYTES BE A CELL THERAPEUTIC STRATEGY FOR CEREBRAL ISCHEMIA?}

Due to the complexity of the excitotoxic effects caused by ischemia/reperfusion, in which pharmacological treatments have only palliative effects or require administration within a short time period after injury (van der Worp and van Gijn, 2007), it is necessary to find alternative therapies focused on long-term neuroprotection. Cell therapy in neuroscience is focused on the search for cells that can induce regeneration, providing a vehicle for corrective molecular systems to trigger endogenous cellular events that can restore tissue homeostasis in progressive neurodegeneration. Currently, many therapeutic strategies using astrocytes have been focused primarily on diseases associated with the spinal cord, such as amyotrophic lateral sclerosis (ALS; Nicaise et al., 2015). In addition, astrocyte transplantation leads to recovery of axonal myelination, modulation of the immune response and release of neurotrophic factors that prevent oxidative stress and excitotoxic damage (Choudhury and Ding, 2015).

Transplantation models have been reported in cerebral ischemia mainly using mesenchymal stem cells (MSC; Hsuan et al., 2016; Lee et al., 2016), induced pluripotent stem cells (iPSCs; Mohamad et al., 2013), and NSCs (Huang et al., 2014; Gervois et al., 2016), showing that there is stimulus of endogenous activity of cells in the injured tissue, generating increased angiogenesis/neovascularization and release of trophic factors, which is chiefly secreted by these cells, allowing the migration to the lesion zone. Likewise, there is axonal recovery, dendritic branching, and synaptogenesis, which are reported mainly surrounding the injury site (Horie et al., 2015). Interestingly, the intravenous NSC administration improves the gliotransmission property, which induced the excitatoryinhibitory balance in the penumbra area (Bacigaluppi et al., 2016).

Other reports have proposed astrocytes as a therapeutic target based on their control by genetic modification of proteins related to the immune response and to the exacerbation of reactivity and cytotoxicity (Choudhury and Ding, 2015; Merienne et al., 2015). We and other researchers have found that Tau hyperphosphorylation is increased by focal and global cerebral ischemia, which is closely related to spatial memory impairment (Gutiérrez-Vargas et al., 2015), implying the alteration of proteins that regulate microtubule assembly, such as glycogen synthase kinase 3 (GSK3; Céspedes-Rubio et al., 2010) and CDK5 (Rashidian et al., 2009; GutiérrezVargas et al., 2016), and remodeling of the actin cytoskeleton, such as the small GTPases RhoA and Rac (Rho family of GTPase members; Posada-Duque et al., 2015b). These proteins have therefore been suggested as key targets in the pathogenesis and recovery of the cerebrovascular unit after infarction (Posada-Duque et al., 2015a, targeting not only neurons but also astrocytes (Posada-Duque et al., 2015b). 


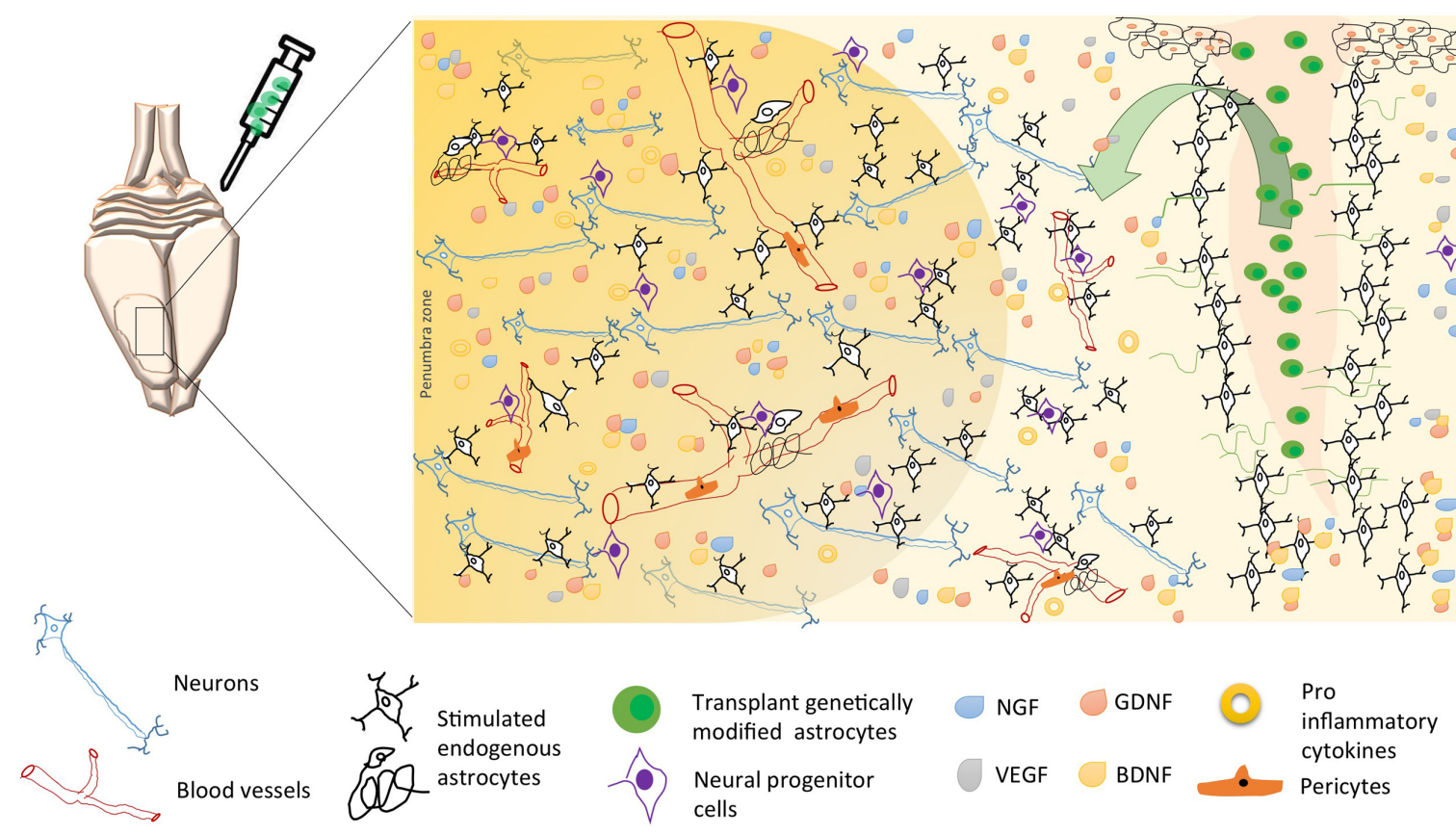

FIGURE 2 | Possible mechanisms of neuroprotection induced by astrocytes' cell therapy after brain stroke. Following brain ischemia, transplantation of genetically modified astrocytes could trigger endogenous neuroprotective mechanisms as neurogenesis, angiogenesis, and regulation of inflammation. These mechanisms are regulated by a paracrine activity, where the astrocyte participates as an intermediary between neurons, endothelial cells, pericytes, microglia, and neural progenitor cells; through the release of neurotrophic factors (BDNF, GDNF, VEGF, GDNF), lower proinflammatory cytokines, and increased reuptake excitatory neurotransmitters, thus avoiding excitotoxicity and, promoting long-term neuronal survival. Arrow, soluble growth factors release by transplanted genetically modified astrocytes ("green cells," e.g., Figure 1C), stimulate endogens astrocytes and endothelial cells, preserving the neurovascular integrity.

In the case of CDK5, we have designed a shRNAmiR that, in a cerebral ischemia model, resulted in neurologic and motor improvement during the first week after ischemia. Additionally, CDK5 RNAi (RNA interference) prevented dysfunctions in learning, memory and reversal learning at a month after ischemia. Interestingly, silencing CDK5 decreases the hyperreactivity of astrocytes at 1 month posttreatment (Gutiérrez-Vargas et al., 2015) but increased astrocytic arborization under neuroprotective conditions at 4 months post-ischemia (Gutiérrez-Vargas et al., 2016). In addition, our in vitro assays have shown that CDK5 RNAi in astrocytes generates stellation and brain-derived neurotrophic factor (BDNF) release in a mechanism dependent on active Rac, providing neuroprotection in co-cultures of astrocytes and neurons (Posada-Duque et al., 2015b). However, it has been widely reported that astrocytes have limited neuroprotective capacity, primarily because of their hypertrophic reactivity under neurotoxic and neurodegenerative processes. This activity involves cytoskeletal changes mediated by Rho GTPases such as Rac/RhoA and by CDK5, as well as the participation of these cells in glial scar formation, the increase of free radicals, and the pro-inflammatory response that prevents neuronal plasticity (Gleichman and Carmichael, 2014). Our most recent findings validated the potential of CDK5 knock-down astrocytes transplanted in ischemic rats, which surprisingly generated motor function recovery, branching of endogenous astrocytes and increased EC adhesion by PECAM1 and Ki67 ${ }^{+}$ cells in SVZ after 1 month post transplantation (BecerraCalixto and Cardona-Gómez, 2016). This functional effect was maintained for 4 months and astrocytes recovery the EC adhesion and transendothelial electrical resistance (TEER) affected by glutamate in a co-culture in vitro model (unpublished data). Therefore, these results lead to the proposal of cell therapy based on silenced astrocytes for genes associated with hyperreactivity (Figure 1C). Based on our evidence, CDK5 knock-down astrocytes may provide neuroprotective functions by inducing the activation of endogenous surrounding astrocytes, which would trigger paracrine signaling or activity of another cell population of the NVU. Transplantation of these modified astrocytes also mobilizes neural progenitor cells, which, combined with their other protective effects, could increase survival and neuroprotection mainly in the penumbra areas of infarcted tissue, recovering neurological function (Figure 2).

\section{CONCLUSION AND PERSPECTIVES}

The field of neuroscience is currently focusing on the study of the function of non-neuronal cells, which seems to play a key role, especially in the survival of neurons upon cerebral injuries. However, it remains highly discussed how and when astrocytes may provide neuroprotection. Several authors indicate that there are different types of astrocytic populations with effects 
on neuroprotection, neurogenesis and regulation of the immune response. Thus, the next challenge for validating the use of astrocytes in cell therapy is to explore the specific profile of neuroprotective astrocytes.

Furthermore, cell therapy has some limitations, such as maintaining long-term restoration, potential regenerative abilities of some types of cells, the capacity of mobilization toward the injured tissue and their safe use in humans. These limitations necessitate further analysis and discussion before the clinical application of those cells. Therefore, further studies must be performed to elucidate how to use astrocytes as targets for cell therapy in acute and chronic neurodegenerative diseases.

\section{REFERENCES}

Abbott, N. J., Rönnbäck, L., and Hansson, E. (2006). Astrocyte-endothelial interactions at the blood-brain barrier. Nat. Rev. Neurosci. 7, 41-53. doi: 10. 1038/nrn1824

Almeida, A., Almeida, J., Bolaños, J. P., and Moncada, S. (2001). Different responses of astrocytes and neurons to nitric oxide: the role of glycolytically generated ATP in astrocyte protection. Proc. Natl. Acad. Sci. U.S.A. 98, 15294-15299. doi: 10.1073/pnas.261560998

Arai, K., and Lo, E. H. (2009). Astrocytes protect oligodendrocyte precursor cells via MEK/ERK and PI3K/Akt signaling. J. Neurosci. Res. 88, 758-763. doi: 10. 1002/jnr.22256

Babaei, S., Teichert-Kuliszewska, K., Zhang, Q., Jones, N., Dumont, D. J., Stewart, D. J., et al. (2003). Angiogenic actions of angiopoietin-1 require endotheliumderived nitric oxide. Am. J. Pathol. 162, 1927-1936. doi: 10.1016/S00029440(10)64326-X

Bacigaluppi, M., Russo, G. L., Peruzzotti-Jametti, L., Rossi, S., Sandrone, S., Butti, E., et al. (2016). Neural stem cell transplantation induces stroke recovery by upregulating glutamate transporter GLT-1 in Astrocytes. J. Neurosci. 36, 10529-10544. doi: 10.1523/JNEUROSCI.1643-16.2016

Bak, L. K., Schousboe, A., and Waagepetersen, H. S. (2006). The glutamate/GABAglutamine cycle: aspects of transport, neurotransmitter homeostasis and ammonia transfer. J. Neurochem. 98, 641-653. doi: 10.1111/j.1471-4159.2006. 03913.x

Bé Langer, M., Allaman, I., and Magistretti, P. J. (2011). Brain energy metabolism: focus on astrocyte-neuron metabolic cooperation. Cell Metab. 14, 724-738. doi: 10.1016/j.cmet.2011.08.016

Bean, B. P. (2007). The action potential in mammalian central neurons. Nat. Rev. Neurosci. 8, 451-465. doi: 10.1038/nrn2148

Becerra-Calixto, A., and Cardona-Gómez, G. P. (2016). Neuroprotection induced by transplanted CDK5 knockdown astrocytes in global cerebral ischemic rats. Mol. Neurobiol. doi: 10.1007/s12035-016-0162-2

Bélanger, M., and Magistretti, P. J. (2009). The role of astroglia in neuroprotection. Dialogues Clin. Neurosci. 11, 281-295.

Bell, R. D., Winkler, E. A., Sagare, A. P., Singh, I., LaRue, B., Deane, R., et al. (2010). Pericytes control key neurovascular functions and neuronal phenotype in the adult brain and during brain aging. Neuron 68, 409-427. doi: 10.1016/j.neuron. 2010.09.043

Belmadani, A., Tran, P. B., Ren, D., and Miller, R. J. (2006). Chemokines regulate the migration of neural progenitors to sites of neuroinflammation. J. Neurosci. 26, 3182-3191. doi: 10.1523/JNEUROSCI.0156-06.2006

Benarroch, E. E. (2005). Neuron-astrocyte interactions: partnership for normal function and disease in the central nervous system. Mayo Clin. Proc. 80, 1326-1338. doi: 10.4065/80.10.1326

Berkich, D. A., Ola, M. S., Cole, J., Sweatt, A. J., Hutson, S. M., and LaNoue, K. F. (2007). Mitochondrial transport proteins of the brain. J. Neurosci. Res. 85, 3367-3377. doi: 10.1002/jnr.21500

Brown, A. M. (2004). Brain glycogen re-awakened. J. Neurochem. 89, 537-552. doi: $10.1111 /$ j.1471-4159.2004.02421.x

\section{FUNDING}

Colciencias \# 111554531400 (2012-2015), Republic bank \# 3494, CODI University of Antioquia 2014-970, Medellín, Colombia.

\section{AUTHOR CONTRIBUTIONS}

$\mathrm{AB}-\mathrm{C}$ wrote the manuscript, GC-G did critical revision of the manuscript for intellectual content. All authors participated in the study concept and design, read and approved the final manuscript.

Brown, A. M., Sickmann, H. M., Fosgerau, K., Lund, T. M., Schousboe, A., Waagepetersen, H. S., et al. (2005). Astrocyte glycogen metabolism is required for neural activity during aglycemia or intense stimulation in mouse white matter. J. Neurosci. Res. 79, 74-80. doi: 10.1002/jnr.20335

Bsibsi, M., Persoon-Deen, C., Verwer, R. W., Meeuwsen, S., Ravid, R., and Van Noort, J. M. (2006). Toll-like receptor 3 on adult human astrocytes triggers production of neuroprotective mediators. Glia 53, 688-695. doi: 10.1002/glia. 20328

Buffo, A., Rite, I., Tripathi, P., Lepier, A., Colak, D., Horn, A. P., et al. (2008). Origin and progeny of reactive gliosis: a source of multipotent cells in the injured brain. Proc. Natl. Acad. Sci. U.S.A. 105, 3581-3586. doi: 10.1073/pnas.0709002105

Caso, J. R., Pradillo, J. M., Hurtado, O., Lorenzo, P., Moro, M. A., and Lizasoain, I. (2007). Toll-like receptor 4 is involved in brain damage and inflammation after experimental stroke. Circulation 115, 1599-1608. doi: 10. 1161/CIRCULATIONAHA.106.603431

Castro, M. A., Beltrán, F. A., Brauchi, S., and Concha, I. I. (2009). A metabolic switch in brain: glucose and lactate metabolism modulation by ascorbic acid. J. Neurochem. 110, 423-440. doi: 10.1111/j.1471-4159.2009.06151.x

Céspedes-Rubio, A., Jurado, F. W., and Cardona-Gómez, G. P. (2010). p120 catenin $/ \alpha \mathrm{N}$-catenin are molecular targets in the neuroprotection and neuronal plasticity mediated by atorvastatin after focal cerebral ischemia. J. Neurosci. Res. 88, 3621-3634. doi: 10.1002/jnr.22511

Chavakis, T., Bierhaus, A., and Nawroth, P. P. (2004). RAGE (receptor for advanced glycation end products): a central player in the inflammatory response. Microbes Infect. 6, 1219-1225. doi: 10.1016/j.micinf.2004.08.004

Chen, Y., and Swanson, R. A. (2003). Astrocytes and brain injury. J. Cereb. Blood Flow Metab. 23, 137-149. doi: 10.1097/01.WCB.0000044631.80210.3C

Chodobski, A., Zink, B. J., and Szmydynger-Chodobska, J. (2011). Blood-brain barrier pathophysiology in traumatic brain injury. Transl. Stroke Res. 2, 492-516. doi: 10.1007/s12975-011-0125-x

Choudhury, G. R., and Ding, S. (2015). Reactive astrocytes and therapeutic potential in focal ischemic stroke. Neurobiol. Dis. 85, 234-244. doi: 10.1016/j. nbd.2015.05.003

Christopherson, K. S., Ullian, E. M., Stokes, C. C. A., Mullowney, C. E., Hell, J. W., Agah, A., et al. (2005). Thrombospondins are astrocyte-secreted proteins that promote CNS synaptogenesis. Cell 120, 421-433. doi: 10.1016/j.cell.2004. 12.020

Cross, M. J., Claesson-Welsh, L., Carmeliet, P., Yancopoulos, G., and Hanahan, D. (2001). FGF and VEGF function in angiogenesis: signalling pathways, biological responses and therapeutic inhibition. Trends Pharmacol. Sci. 22, 201-207. doi: 10.1016/S0165-6147(00)01676-X

Dallérac, G., Chever, O., and Rouach, N. (2013). How do astrocytes shape synaptic transmission? Insights from electrophysiology. Front. Cell Neurosci. 7:159. doi: 10.3389/fncel.2013.00159

D'Ambrosio, R., Gordon, D. S., and Winn, H. R. (2002). Differential Role of KIR Channel and $\mathrm{Na}+/ \mathrm{K}+-$ Pump in the Regulation of Extracellular $\mathrm{K}+$ in Rat Hippocampus. J. Neurophysiol 87, 87-102.

Danbolt, N. C. (2001). Glutamate uptake. Prog. Neurobiol. 65, 1-105. doi: 10.1016/ S0301-0082(00)00067-8 
de Pablo, Y., Nilsson, M., Pekna, M., and Pekny, M. (2013). Intermediate filaments are important for astrocyte response to oxidative stress induced by oxygen-glucose deprivation and reperfusion. Histochem. Cell Biol. 140, 81-91. doi: $10.1007 / \mathrm{s} 00418-013-1110-0$

Dong, Y., and Benveniste, E. N. (2001). Immune function of astrocytes. Glia 36, 180-190. doi: 10.1002/glia.1107

Dringen, R. (2000). Metabolism and functions of glutathione in brain. Prog. Neurobiol. 62, 649-671. doi: 10.1016/S0301-0082(99)00060-X

Duan, C. L., Liu, C. W., Shen, S. W., Yu, Z., Mo, J. L., Chen, X. H., et al. (2015). Striatal astrocytes transdifferentiate into functional mature neurons following ischemic brain injury. Glia 63, 1660-1670. doi: 10.1002/glia.22837

Eng, L. F., Ghirnikar, R. S., and Lee, Y. L. (2000). Glial fibrillary acidic protein: GFAP-thirty-one years (1969-2000). Neurochem. Res. 25, 1439-1451. doi: 10. 1023/A:1007677003387

Faiz, M., Sachewsky, N., Gascón, S., Bang, K. W. A., Morshead, C. M., and Nagy, A. (2015). Adult neural stem cells from the subventricular zone give rise to reactive astrocytes in the cortex after stroke. Cell Stem Cell 17, 624-634. doi: 10.1016/j. stem.2015.08.002

Farina, C., Aloisi, F., and Meinl, E. (2007). Astrocytes are active players in cerebral innate immunity. Trends Immunol. 28, 138-145. doi: 10.1016/j.it.2007.01.005

Garcia-Nogales, P., Almeida, A., and Bolanos, J. P. (2003). Peroxynitrite protects neurons against nitric oxide-mediated apoptosis: a key role for glucose-6phosphate dehydrogenase activity in neuroprotection. J. Biol. Chem. 278, 864-874. doi: 10.1074/jbc.M206835200

Gervois, P., Wolfs, E., Ratajczak, J., Dillen, Y., Vangansewinkel, T., Hilkens, P., et al. (2016). Stem cell-based therapies for ischemic stroke: preclinical results and the potential of imaging-assisted evaluation of donor cell fate and mechanisms of brain regeneration. Med. Res. Rev. 36, 1080-1126. doi: 10.1002/med.21400

Gibbs, M., Anderson, D., and Hertz, L. (2006). Inhibition of glycogenolysis in astrocytes interrupts memory consolidation in young chickens. Glia. 54, 214-222. doi: 10.1002/glia.20377

Gleichman, A. J., and Carmichael, S. T. (2014). Astrocytic therapies for neuronal repair in stroke. Neurosci. Lett. 565C, 47-52. doi: 10.1016/j.neulet.2013.10.055

Gorina, R., Font-Nieves, M., Márquez-Kisinousky, L., Santalucia, T., and Planas, A. M. (2011). Astrocyte TLR4 activation induces a proinflammatory environment through the interplay between MyD88-dependent NFкB signaling, MAPK, and Jak1/Statl pathways. Glia 59, 242-255. doi: 10.1002/glia.21094

Goss, J. R., O’Malley, M. E., Zou, L., Styren, S. D., Kochanek, P. M., and DeKosky, S. T. (1998). Astrocytes are the major source of nerve growth factor upregulation following traumatic brain injury in the rat. Exp. Neurol. 149, 301-309. doi: 10.1006/exnr.1997.6712

Götz, M., Sirko, S., Beckers, J., and Irmler, M. (2015). Reactive astrocytes as neural stem or progenitor cells: In vivo lineage, In vitro potential, and Genome-wide expression analysis. Glia 63, 1452-1468. doi: 10.1002/glia.22850

Grant, P., Sharma, P., and Pant, H. C. (2001). Cyclin-dependent protein kinase 5 (Cdk5) and the regulation of neurofilament metabolism. Eur. J. Biochem. 268, 1534-1546. doi: 10.1046/j.1432-1327.2001.02025.x

Gutiérrez-Vargas, J. A., Moreno, H., and Cardona-Gómez, G. P. (2016). Targeting CDK5 post-stroke provides long-term neuroprotection and rescues synaptic plasticity. J. Cereb. Blood Flow Metab. doi: 10.1177/0271678X16662476 [Epub ahead of print].

Gutiérrez-Vargas, J. A., Múnera, A., and Cardona-Gómez, G. P. (2015). CDK5 knockdown prevents hippocampal degeneration and cognitive dysfunction produced by cerebral ischemia. J. Cereb. Blood Flow Metab. 35, 1937-1949. doi: $10.1038 /$ jcbfm.2015.150

Hayakawa, K., Pham, L. D., Katusic, Z. S., Arai, K., and Lo, E. H. (2012). Astrocytic high-mobility group box 1 promotes endothelial progenitor cell-mediated neurovascular remodeling during stroke recovery. Proc. Natl. Acad. Sci. U.S.A. 109, 7505-7510. doi: 10.1073/pnas.1121146109

Hayakawa, K., Pham, L.-D. D., Som, A. T., Lee, B. J., Guo, S., Lo, E. H., et al. (2011). Vascular endothelial growth factor regulates the migration of oligodendrocyte precursor cells. J. Neurosci. 31, 10666-10670. doi: 10.1523/JNEUROSCI.194411.2011

He, Y., Li, H. L., Xie, W. Y., Yang, C. Z., Yu, A. C., and Wang, Y. (2007). The presence of active Cdk5 associated with p35 in astrocytes and its important role in process elongation of scratched astrocyte. Glia 55, 573-583. doi: 10.1002/glia. 20485
Herrero-Mendez, A., Almeida, A., Fernández, E., Maestre, C., Moncada, S., and Bolaños, J. P. (2009). The bioenergetic and antioxidant status of neurons is controlled by continuous degradation of a key glycolytic enzyme by APC/CCdh1. Nat. Cell Biol. 11, 747-752. doi: 10.1038/ncb1881

Herx, L. M., Rivest, S., and Yong, V. W. (2000). Central nervous systeminitiated inflammation and neurotrophism in trauma: IL-1 beta is required for the production of ciliary neurotrophic factor. J. Immunol. 165, 2232-2239. doi: 10.4049/jimmunol.165.4.2232

Horie, N., Hiu, T., and Nagata, I. (2015). Stem cell transplantation enhances endogenous brain repair after experimental stroke. Neurol. Med. Chir. 55 (Suppl. 1), 107-112. doi: 10.2176/nmc.ra.2014-0271

Hsuan, Y. C.-Y., Lin, C.-H., Chang, C.-P., and Lin, M.-T. (2016). Mesenchymal stem cell-based treatments for stroke, neural trauma, and heat stroke. Brain Behav. 6:e00526. doi: 10.1002/brb3.526

$\mathrm{Hu}$, J., and Van Eldik, L. J. (1999). Glial-derived proteins activate cultured astrocytes and enhance beta amyloid-induced glial activation. Brain Res. 842, 46-54. doi: 10.1016/S0006-8993(99)01804-1

Huang, L., Wong, S., Snyder, E. Y., Hamblin, M. H., and Lee, J. P. (2014). Human neural stem cells rapidly ameliorate symptomatic inflammation in early-stage ischemic-reperfusion cerebral injury. Stem Cell Res. Ther. 5, 129. doi: 10.1186/ scrt519

Igarashi, Y., Utsumi, H., Chiba, H., Yamada-Sasamori, Y., Tobioka, H., Kamimura, Y., et al. (1999). Glial cell line-derived neurotrophic factor induces barrier function of endothelial cells forming the blood-brain barrier. Biochem. Biophys. Res. Commun. 261, 108-112. doi: 10.1006/bbrc.1999.0992

Jiang, P., Chen, C., Wang, R., Chechneva, O. V., Chung, S. H., Rao, M. S., et al. (2013). hESC-derived Olig2+ progenitors generate a subtype of astroglia with protective effects against ischaemic brain injury. Nat. Commun. 4, 2196. doi: $10.1038 /$ ncomms3196

Kaczor, P., Rakus, D., and Mozrzymas, J. W. (2015). Neuron-astrocyte interaction enhance GABAergic synaptic transmission in a manner dependent on key metabolic enzymes. Front. Cell Neurosci. 9:120. doi: 10.3389/fncel.2015.00120

Kitchen, P., Day, R. E., Taylor, L. H., Salman, M. M., Bill, R. M., Conner, M. T., et al. (2015). Identification and molecular mechanisms of the rapid tonicity-induced relocalization of the aquaporin 4 channel. J. Biol. Chem. 290, 16873-16881. doi: 10.1074/jbc.M115.646034

Krum, J. M., and Rosenstein, J. M. (1998). VEGF mRNA and its receptor flt-1 are expressed in reactive astrocytes following neural grafting and tumor cell implantation in the adult CNS. Exp. Neurol. 154, 57-65. doi: 10.1006/exnr.1998. 6930

Laywell, E. D., Rakic, P., Kukekov, V. G., Holland, E. C., and Steindler, D. A. (2000). Identification of a multipotent astrocytic stem cell in the immature and adult mouse brain. Proc. Natl. Acad. Sci. U. S. A. 97, 13883-13888. doi: $10.1073 /$ pnas. 250471697

Le Bras, B., Barallobre, M.-J., Homman-Ludiye, J., Ny, A., Wyns, S., Tammela, T., et al. (2006). VEGF-C is a trophic factor for neural progenitors in the vertebrate embryonic brain. Nat. Neurosci. 9, 340-348. doi: 10.1038/nn1646

Lebkuechner, I., Wilhelmsson, U., Möllerström, E., Pekna, M., and Pekny, M. (2015). Heterogeneity of Notch signaling in astrocytes and the effects of GFAP and vimentin deficiency. J. Neurochem. 135, 234-248. doi: 10.1111/jnc. 13213

Lee, J. Y., Kim, E., Choi, S.-M., Kim, D.-W., Kim, K. P., Lee, I., et al. (2016). Microvesicles from brain-extract-treated mesenchymal stem cells improve neurological functions in a rat model of ischemic stroke. Sci. Rep. 6:33038. doi: 10.1038/srep33038

Li, L., Lundkvist, A., Andersson, D., Wilhelmsson, U., Nagai, N., Pardo, A. C., et al. (2008). Protective role of reactive astrocytes in brain ischemia. J. Cereb. Blood Flow Metab. 28, 468-481. doi: 10.1038/sj.jcbfm.9600546

Li, M., Sun, L., Li, Y., Xie, C., Wan, D., and Luo, Y. (2014). Oxygen glucose deprivation/reperfusion astrocytes promotes primary neural stem/progenitor cell proliferation by releasing high-mobility group box 1. Neurochem. Res. 39, 1440-1450. doi: 10.1007/s11064-014-1333-z

Liang, J., Takeuchi, H., Doi, Y., Kawanokuchi, J., Sonobe, Y., Jin, S., et al. (2008). Excitatory amino acid transporter expression by astrocytes is neuroprotective against microglial excitotoxicity. Brain Res. 1210, 11-19. doi: 10.1016/j.brainres. 2008.03.012

Lichter-Konecki, U. (2008). Profiling of astrocyte properties in the hyperammonaemic brain: shedding new light on the pathophysiology of 
the brain damage in hyperammonaemia. J. Inherit. Metab. Dis. 31, 492-502. doi: 10.1007/s10545-008-0834-9

Liebl, J., Fürst, R., Vollmar, A. M., and Zahler, S. (2011). Twice switched at birth: cell cycle-independent roles of the "neuron-specific" cyclin-dependent kinase 5 (Cdk5) in non-neuronal cells. Cell. Signal. 23, 1698-1707. doi: 10.1016/j.cellsig. 2011.06.020

Lipton, P. (1999). Ischemic cell death in brain neurons. Physiol. Rev. 79, 1431-1568.

Liu, R., Tian, B., Gearing, M., Hunter, S., Ye, K., and Mao, Z. (2008). Cdk5mediated regulation of the PIKE-A-Akt pathway and glioblastoma cell invasion. Proc. Natl. Acad. Sci. U.S.A. 105, 7570-7575. doi: 10.1073/pnas.0712306105

Liu, W., Tang, Y., and Feng, J. (2011). Cross talk between activation of microglia and astrocytes in pathological conditions in the central nervous system. Life Sci. 89, 141-146. doi: 10.1016/j.lfs.2011.05.011

Lovatt, D., Sonnewald, U., Waagepetersen, H. S., Schousboe, A., He, W., Lin, J. H.-C., et al. (2007). The transcriptome and metabolic gene signature of protoplasmic astrocytes in the adult murine cortex. J. Neurosci. 27, 12255-12266. doi: 10.1523/JNEUROSCI.3404-07.2007

Magnusson, J. P., and Frisén, J. (2016). Stars from the darkest night: unlocking the neurogenic potential of astrocytes in different brain regions. Development 143 , 1075-1086. doi: 10.1242/dev.133975

Maier, B., Laurer, H.-L., Rose, S., Buurman, W. A., and Marzi, I. (2005). Physiological levels of pro- and anti-inflammatory mediators in cerebrospinal fluid and plasma: a normative study. J. Neurotrauma 22, 822-835. doi: 10.1089/ neu.2005.22.822

Mason, J. L., Suzuki, K., Chaplin, D. D., and Matsushima, G. K. (2001). Interleukinlbeta promotes repair of the CNS. J. Neurosci. 21, 7046-7052.

Merienne, N., Delzor, A., Viret, A., Dufour, N., Rey, M., Hantraye, P., et al. (2015). Gene transfer engineering for astrocyte-specific silencing in the CNS. Gene Ther. 22, 830-839. doi: 10.1038/gt.2015.54

Mohamad, O., Drury-Stewart, D., Song, M., Faulkner, B., Chen, D., Yu, S. P., et al. (2013). Vector-free and transgene-free human iPS cells differentiate into functional neurons and enhance functional recovery after ischemic stroke in mice. PLoS ONE 8:e64160. doi: 10.1371/journal.pone.0064160

Mohn, T. C., and Koob, A. O. (2015). Adult astrogenesis and the etiology of cortical neurodegeneration. J. Exp. Neurosci. 9, 25-34. doi: 10.4137/JEN.S25520

Morganti-Kossmann, M. C., Satgunaseelan, L., Bye, N., and Kossmann, T. (2007). Modulation of immune response by head injury. Injury 38, 1392-1400. doi: 10.1016/j.injury.2007.10.005

Mori, T., Buffo, A., and Götz, M. (2005). The novel roles of glial cells revisited: the contribution of radial glia and astrocytes to neurogenesis. Curr. Top. Dev. Biol. 69, 67-99. doi: 10.1016/S0070-2153(05)69004-7

Mori, T., Koyama, N., Arendash, G. W., Horikoshi-Sakuraba, Y., Tan, J., and Town, T. (2009). Overexpression of human S100B exacerbates cerebral amyloidosis and gliosis in the Tg2576 mouse model of Alzheimer's disease. Glia 58, 300-314. doi: 10.1002/glia.20924

Nato, G., Caramello, A., Trova, S., and Avataneo, V. (2015). Striatal astrocytes produce neuroblasts in an excitotoxic model of Huntington's disease. Development 142, 840-845. doi: 10.1242/dev.116657

Newman, E. A. (2015). Glial cell regulation of neuronal activity and blood flow in the retina by release of gliotransmitters. Philos. Trans. R. Soc. Lond. B Biol. Sci. 370:20140195. doi: 10.1098/rstb.2014.0195

Nicaise, C., Mitrecic, D., Falnikar, A., and Lepore, A. C. (2015). Transplantation of stem cell-derived astrocytes for the treatment of amyotrophic lateral sclerosis and spinal cord injury. World J. Stem Cells 7, 380-398. doi: 10.4252/wjsc.v7. i 2.380

Ohab, J. J., Fleming, S., Blesch, A., and Carmichael, S. T. (2006). A Neurovascular niche for neurogenesis after stroke. J. Neurosci. 26, 1300-1307. doi: 10.1523/ JNEUROSCI.4323-06.2006

Oliveira, S. L., Pillat, M. M., Cheffer, A., Lameu, C., Schwindt, T. T., and Ulrich, H. (2013). Functions of neurotrophins and growth factors in neurogenesis and brain repair. Cytom. A 83, 76-89. doi: 10.1002/cyto.a.22161

Pekny, M., and Nilsson, M. (2005). Astrocyte activation and reactive gliosis. Glia 50, 427-434. doi: 10.1002/glia.20207

Pentreath, V., and Slamon, N. (2000). Astrocyte phenotype and prevention against oxidative damage in neurotoxicity. Hum. Exp. Toxicol. 19, 641-649. doi: 10. 1191/096032700676221595

Piani, D., Frei, K., Pfister, H.-W., and Fontana, A. (1993). Glutamate uptake by astrocytes is inhibited by reactive oxygen intermediates but not by other macrophage-derived molecules including cytokines, leukotrienes or plateletactivating factor. J. Neuroimmunol. 48, 99-104. doi: 10.1016/0165-5728(93) 90063-5

Posada-Duque, R. A., López-Tobón, A., Piedrahita, D., González-Billault, C., and Cardona-Gomez, G. P. (2015a). p35 and Rac1 underlie the neuroprotection and cognitive improvement induced by CDK5 silencing. J. Neurochem. 134, 354-370. doi: 10.1111/jnc.13127

Posada-Duque, R. A., Palacio-Castañeda, V., and Cardona-Gómez, G. P. (2015b). CDK5 knockdown in astrocytes provide neuroprotection as a trophic source via Rac1. Mol. Cell. Neurosci. 68, 151-166. doi: 10.1016/j.mcn.2015.07.001

Rashidian, J., Rousseaux, M. W., Venderova, K., Qu, D., Callaghan, S. M., Phillips, M., et al. (2009). Essential role of cytoplasmic cdk5 and Prx2 in multiple ischemic injury models, in vivo. J. Neurosci. 29, 12497-12505. doi: 10.1523/ JNEUROSCI.3892-09.2009

Robel, S., Buckingham, S. C., Boni, J. L., Campbell, S. L., Danbolt, N. C., Riedemann, T., et al. (2015). Reactive astrogliosis causes the development of spontaneous seizures. J. Neurosci. 35, 3330-3345. doi: 10.1523/JNEUROSCI. 1574-14.2015

Rodríguez, J. J., Olabarria, M., Chvatal, A., and Verkhratsky, A. (2009). Astroglia in dementia and Alzheimer's disease. Cell Death Differ. 16, 378-385. doi: 10.1038/ cdd.2008.172

Rostworowski, M., Balasingam, V., Chabot, S., Owens, T., and Yong, V. W. (1997). Astrogliosis in the neonatal and adult murine brain post-trauma: elevation of inflammatory cytokines and the lack of requirement for endogenous interferon-

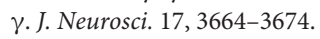

Rz, P. M., Heese, K., Dimitriades-Schmutz, B., Rose-John, S., and Otten, U. (1999). Role of interleukin-6 and soluble IL-6 receptor in region-specific induction of astrocytic differentiation and neurotrophin expression. Glia 26, 191-200. doi: 10.1002/(SICI) 1098-1136(199905)26:3<191::AID-GLIA1>3.0.CO;2-\#

Schwartz, J. P., and Nishiyama, N. (1994). Neurotrophic factor gene expression in astrocytes during development and following injury. Brain Res. Bull. 35, 403-407. doi: 10.1016/0361-9230(94)90151-1

Seri, B., García-Verdugo, J. M., McEwen, B. S., and Alvarez-Buylla, A. (2001). Astrocytes give rise to new neurons in the adult mammalian hippocampus. J. Neurosci. 21, 7153-7160.

Shen, J., Petersen, K. F., Behar, K. L., Brown, P., Nixon, T. W., Mason, G. F., et al. (1999). Determination of the rate of the glutamate/glutamine cycle in the human brain by in vivo 13C NMR. Proc. Natl. Acad. Sci. U.S.A. 96, 8235-8240. doi: 10.1073/PNAS.96.14.8235

Shen, L. H., Li, Y., and Chopp, M. (2010). Astrocytic endogenous glial cell derived neurotrophic factor production is enhanced by bone marrow stromal cell transplantation in the ischemic boundary zone after stroke in adult rats. Glia 58, 1074-1081. doi: 10.1002/glia.20988

Shen, S.-W., Duan, C.-L., Chen, X.-H., Wang, Y.-Q., Sun, X., Zhang, Q.-W., et al. (2016). Neurogenic effect of VEGF is related to increase of astrocytes transdifferentiation into new mature neurons in rat brains after stroke. Neuropharmacology 108, 451-461. doi: 10.1016/j.neuropharm.2015. 11.012

Shen, Y., Sun, A., Wang, Y., Cha, D., Wang, H., Wang, F., et al. (2012). Upregulation of mesencephalic astrocyte-derived neurotrophic factor in glial cells is associated with ischemia-induced glial activation. J. Neuroinflamm. 9:254. doi: 10.1186/1742-2094-9-254

Shih, A. Y., Li, P., and Murphy, T. H. (2005). A small-molecule-inducible Nrf2mediated antioxidant response provides effective prophylaxis against cerebral ischemia in vivo. J. Neurosci. 25, 10321-10335. doi: 10.1523/JNEUROSCI.401405.2005

Shimada, I. S., Borders, A., Aronshtam, A., and Spees, J. L. (2011). Proliferating reactive astrocytes are regulated by notch-1 in the peri-infarct area after stroke. Stroke 42, 3231-3237. doi: 10.1161/STROKEAHA.111.623280

Silver, J., and Miller, J. H. (2004). Regeneration beyond the glial scar. Nat. Rev. Neurosci. 5, 146-156. doi: 10.1038/nrn1326

Sofroniew, M. V. (2014). Multiple roles for astrocytes as effectors of cytokines and inflammatory mediators. Neuroscientist 20, 160-172. doi: 10.1177/ 1073858413504466

Sofroniew, M. V. (2015). Astrocyte barriers to neurotoxic inflammation. Nat. Rev. Neurosci. 16, 249-263. doi: 10.1038/nrn3898

Sofroniew, M. V., and Vinters, H. V. (2010). Astrocytes: biology and pathology. Acta Neuropathol. 119, 7-35. doi: 10.1007/s00401-009-0619-8 
Sultan, S., Li, L., Moss, J., Petrelli, F., Cassé, F., Gebara, E., et al. (2015). Synaptic integration of adult-born hippocampal neurons is locally controlled by astrocytes. Neuron $88,957-972$. doi: 10.1016/j.neuron.2015.10.037

Sun, D., Lye-Barthel, M., Masland, R. H., and Jakobs, T. C. (2010). Structural remodeling of fibrous astrocytes after axonal injury. J. Neurosci. 30, 14008-14019. doi: 10.1523/JNEUROSCI.3605-10.2010

Susarla, B. T. S., Villapol, S., Yi, J., Geller, H. M., and Symes, A. J. (2014). Temporal patterns of cortical proliferation of glial cell populations after traumatic brain injury in mice. ASN Neuro 6, 159-170. doi: 10.1042/AN20130034

Suzuki, A., Stern, S. A., Bozdagi, O., Huntley, G. W., Walker, R. H., Magistretti, P. J., et al. (2011). Astrocyte-neuron lactate transport is required for long-term memory formation. Cell 144, 810-823. doi: 10.1016/j.cell.2011.02.018

Suzumura, A., Takeuchil, H., Zhang, G., Kuno, R., and Mizuno, T. (2006). Roles of glia-derived cytokines on neuronal degeneration and regeneration. Ann. N. Y. Acad. Sci. 1088, 219-229. doi: 10.1196/annals.1366.012

Swanson, R. A., Ying, W., and Kauppinen, T. M. (2004). Astrocyte influences on ischemic neuronal death. Curr. Mol. Med. 4, 193-205. doi: 10.2174/ 1566524043479185

Tao, Y., Ma, L., Liao, Z., Le, Q., Yu, J., Liu, X., et al. (2015). Astroglial $\beta$-Arrestin1mediated nuclear signaling regulates the expansion of neural precursor cells in adult Hippocampus. Sci. Rep. 5:15506. doi: 10.1038/srep15506

Theodoric, N., Bechberger, J. F., Naus, C. C., and Sin, W. C. (2012). Role of gap junction protein connexin 43 in astrogliosis induced by brain injury. PLOS ONE 7:e47311. doi: 10.1371/journal.pone.0047311

Tran, P. B., and Miller, R. J. (2003). Chemokine receptors: signposts to brain development and disease. Nat. Rev. Neurosci. 4, 444-455. doi: 10.1038/ nrn1116

van der Worp, H. B., and van Gijn, J. (2007). Clinical practice. Acute ischemic stroke. N. Engl. J. Med. 357, 572-579. doi: 10.1056/NEJMcp072057

Walz, W. (2000). Role of astrocytes in the clearance of excess extracellular potassium. Neurochem. Int. 36, 291-300. doi: 10.1016/S0197-0186(99)00137-0

Wuestefeld, R., Chen, J., Meller, K., Brand-Saberi, B., and Theiss, C. (2012). Impact of vegf on astrocytes: analysis of gap junctional intercellular communication, proliferation, and motility. Glia 60, 936-947. doi: 10.1002/glia.22325
Xin, H., Li, Y., Shen, L. H., Liu, X., Wang, X., Zhang, J., et al. (2010). Increasing tPA activity in astrocytes induced by multipotent mesenchymal stromal cells facilitate neurite outgrowth after stroke in the mouse. PLOS ONE 5:e9027. doi: 10.1371/journal.pone.0009027

Yang, T., Sun, Y., and Zhang, F. (2016). "The role of nonneuronal Nrf2 pathway in ischemic stroke: damage control and potential tissue repair," in Non-Neuronal Mechanisms of Brain Damage and Repair After Stroke, eds J. Chen, J. H. Zhang, and X. Hu (Cham: Springer International Publishing), 377-397. doi: 10.1007/ 978-3-319-32337-4_18

Yi, J.-H., and Hazell, A. S. (2006). Excitotoxic mechanisms and the role of astrocytic glutamate transporters in traumatic brain injury. Neurochem. Int. 48, 394-403. doi: 10.1016/j.neuint.2005.12.001

Zeng, X.-N., Sun, X.-L., Gao, L., Fan, Y., Ding, J.-H., and Hu, G. (2007). Aquaporin4 deficiency down-regulates glutamate uptake and GLT-1 expression in astrocytes. Mol. Cell. Neurosci. 34, 34-39. doi: 10.1016/j.mcn.2006.09.008

Zou, J., Wang, Y.-X., Dou, F.-F., Lü, H.-Z., Ma, Z.-W., Lu, P.-H., et al. (2010). Glutamine synthetase down-regulation reduces astrocyte protection against glutamate excitotoxicity to neurons. Neurochem. Int. 56, 577-584. doi: 10.1016/ j.neuint.2009.12.021

Conflict of Interest Statement: The authors declare that the research was conducted in the absence of any commercial or financial relationships that could be construed as a potential conflict of interest.

The reviewer PFC and handling Editor declared their shared affiliation, and the handling Editor states that the process nevertheless met the standards of a fair and objective review.

Copyright (c) 2017 Becerra-Calixto and Cardona-Gómez. This is an open-access article distributed under the terms of the Creative Commons Attribution License (CC BY). The use, distribution or reproduction in other forums is permitted, provided the original author(s) or licensor are credited and that the original publication in this journal is cited, in accordance with accepted academic practice. No use, distribution or reproduction is permitted which does not comply with these terms. 